Med Klin Intensivmed Notfmed 2013.

108:144-148

DOI 10.1007/s00063-012-0184-0

Received: 7 April 2012

Revised: 11 September 2012

Accepted: 25 September 2012

Published online: 8. November 2012

○) Springer-Verlag Berlin Heidelberg 2012

\section{Redaktion}

R. Riessen, Tübingen

\author{
A. Abdel Samie ${ }^{1}$ R. Sun ${ }^{1}$ A. Huber ${ }^{1}$ W. Höpfner ${ }^{2}$ L. Theilmann ${ }^{1}$ \\ ${ }^{1}$ Department of Gastroenterology, Pforzheim Hospital, Pforzheim \\ ${ }^{2}$ Institute for Radiology and Nuclear Medicine, Pforzheim Hospital, Pforzheim
}

\title{
Spontaneous intramural small-bowel hematoma secondary to anticoagulant therapy: a case series
}

$[7,8,9]$. The incidence of this clinical entity is predicted to further increase as a result of the wide use of long-term anticoagulation in an aging population.

\section{Patients and methods}

\section{Case 1} unusual ways.

Intramural small-bowel hematoma has been recognized for many years as a complication of blunt trauma, especially in children. In contrast to traumatic small-bowel hematoma, which mainly affects the duodenum and tends to involve a short intestinal segment, spontaneous small-bowel hematoma is more extensive and most commonly involves the jejunum, followed by the ileum and duodenum [1]. The most common cause of this condition is over-anticoagulation under phenprocoumon/warfarin.

Small-bowel hematoma was initially reported by McLauchlan in 1838 at the autopsy of a 49-year-old man who died of dehydration and duodenal obstruction resulting from a false aneurysmal tumor occluding the duodenum [2].

About 70 years later, Sutherland described a case of a nontraumatic intramural small-bowel hematoma in a child with Henoch-Schönlein purpura presenting with intussusception [3]. Hematoma of the esophagus, colon, and rectum has also been reported $[4,5,6]$.

Nontraumatic spontaneous intramural small-bowel hematoma, once considered a rare complication of anticoagulation, is being reported with increasing frequency
An 80-year-old woman was admitted to hospital owing to jaundice, upper abdominal pain, nausea, and vomiting. The patient was on phenprocoumon because of chronic atrial fibrillation. Physical examination showed right upper quadrant tenderness and jaundice.

Laboratory studies revealed abnormal coagulation test results with a prolonged prothrombin time (international normalized ratio, INR >6.0) and a prolonged activated partial thromboplastin time of more than 110 s. Furthermore, her liver test results were abnormal with a total bilirubin of $12 \mathrm{mg} / \mathrm{dl}$, alanine aminotransfersonography demonstrating a heterogeneous mass within the duodenal wall with duodenal wall thickening (arrow)
Fig. $1>$ Abdominal ase at $170 \mathrm{U} / \mathrm{l}$, aspartate aminotransferase at $230 \mathrm{U} / \mathrm{l}$, and alkaline phosphatase at $1,050 \mathrm{U} / \mathrm{l}$. The white blood cell count was $16 / \mathrm{nl}$ and hemoglobin was $10 \mathrm{~g} / \mathrm{dl}$ with a normocytic anemia blood picture.

Abdominal ultrasonography (US) demonstrated a heterogeneous mass within the duodenal wall with duodenal wall thickening (- Fig. 1, arrow) and dilatation of both bile and pancreatic ducts.

Noncontrast computed tomography (CT; - Fig. 2, arrow) confirmed the sonographic findings, showing circumferential wall thickening and luminal narrowing of the descending duodenum consistent with intramural hematoma. On magnetic resonance imaging (MRI) (• Fig. 3, arrow) a well-defined concentric ring configuration (ring sign) was detected, a finding which helped to confirm the diagnosis.

Phenprocoumon therapy was stopped and the patient was treated conservatively with vitamin $\mathrm{K}$ and fresh frozen plasma.

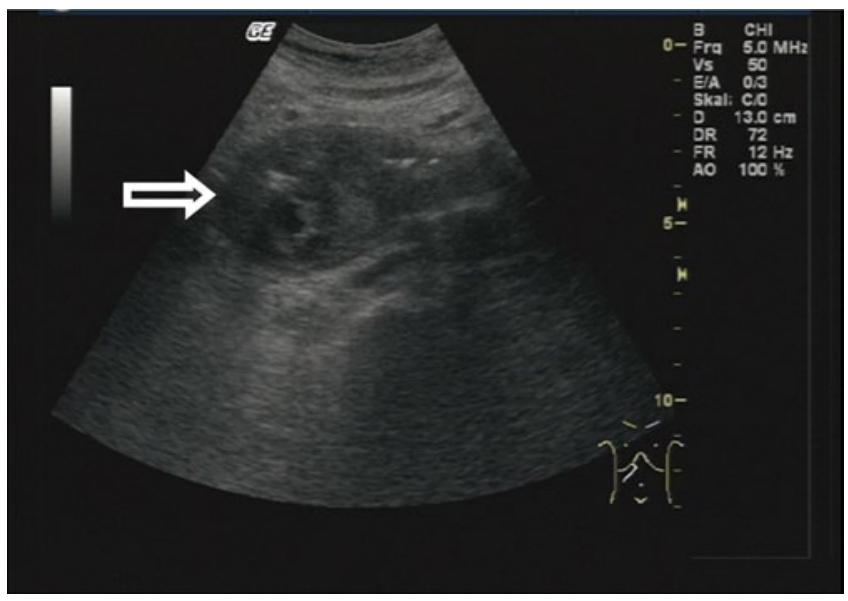




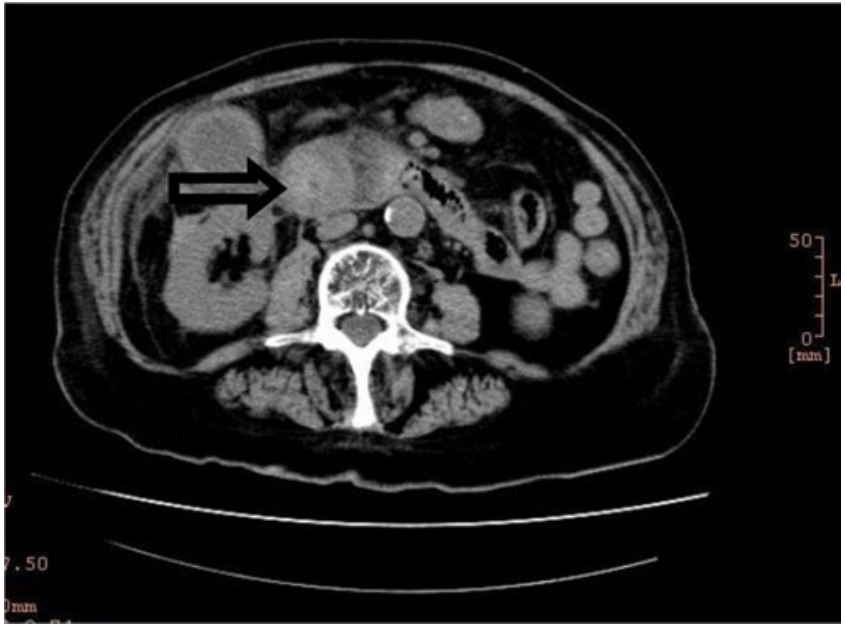

Fig. $2 \Delta$ Noncontrast computed tomography showing circumferential wall thickening and luminal narrowing of the descending duodenum (arrow)

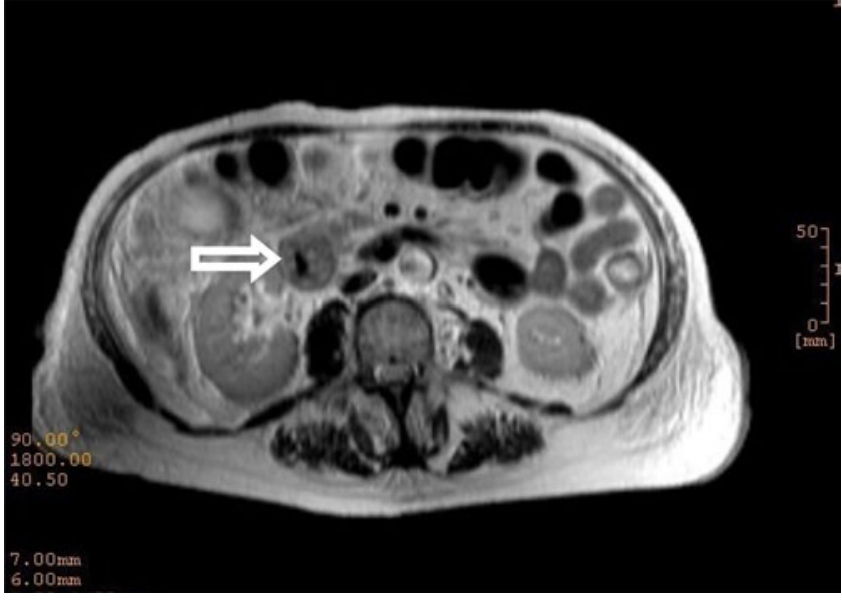

Fig. $3 \Delta$ Magnetic resonance image showing a well-defined concentric ring configuration (ring sign, arrow)

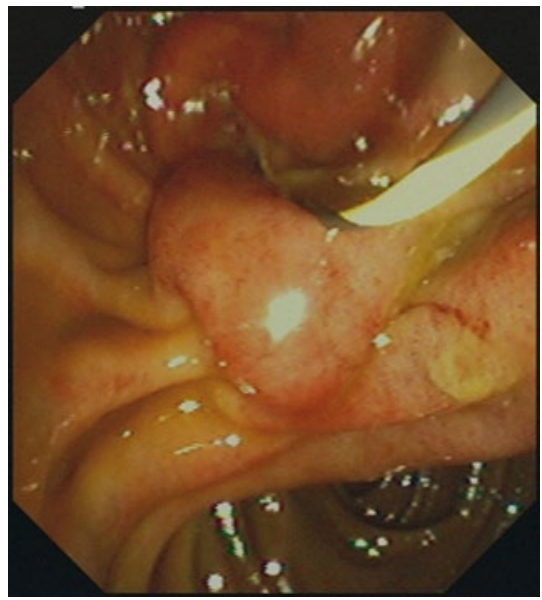

Fig. $4 \Delta$ Endoscopic retrograde cholangiopancreatography showing duodenal wall hematoma compressing the biliary tract

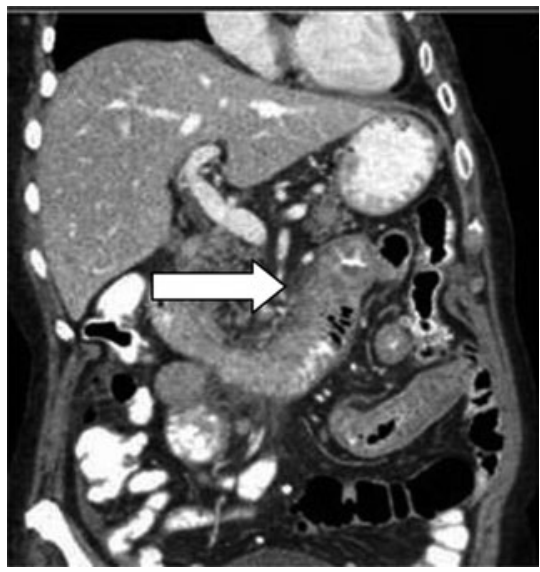

Fig. $6 \Delta$ Contrast-enhanced CT demonstrating circumferential wall thickening with lumen narrowing of the proximal jejunum (arrow)

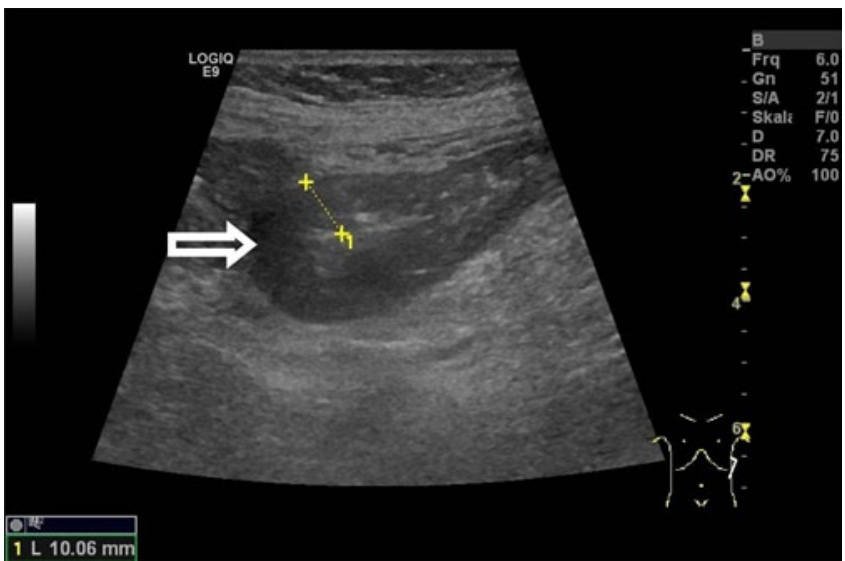

Fig. $5<$ Ultrasonography showing a heterogeneous thickening of the proximal jejunum (arrow)
Endoscopic retrograde cholangiopancreatography (• Fig. 4) was performed showing a duodenal wall hematoma compressing the biliary tract and confirming the noninvasive imaging findings.

Obstructive symptoms resolved under conservative therapy.

\section{Case 2}

An 85-year-old woman was admitted to our unit owing to abdominal pain, nausea, and vomiting that had started 2 days earlier. The patient was under anticoagulation with phenprocoumon because of atrial fibrillation.

Laboratory tests revealed prolonged prothrombin time (INR $>6$ ) and activated partial thromboplastin time (81 s), nor- mocytic anemia (hemoglobin $8.9 \mathrm{~g} / \mathrm{dl}$ ), and leukocytosis (13/nl).

US showed a heterogeneous thickening of the proximal jejunum (• Fig. 5).

Contrast-enhanced CT demonstrated circumferential wall thickening with a narrowing lumen of the proximal jejunum (• Fig. 6).

Both the US and the CT findings were suggestive of intramural hematoma of the proximal jejunum leading to intestinal obstruction.

Phenprocoumon was stopped and two units of packed red blood cells and vitamin $\mathrm{K}$ were administered. Conservative therapy proved successful and complete resolution of obstructive symptoms was achieved. 


\section{Case 3}

A 73-year-old man was admitted to hospital suffering from abdominal pain and melena for about 4 days. The patient was on phenprocoumon because of atrial fibrillation.

Clinical examination revealed abdominal distension and diffuse tenderness of the abdomen. Abnormal laboratory results were as follows: hemoglobin, $11.2 \mathrm{~g} / \mathrm{dl}$; white cell count, $16.5 / \mathrm{nl}$; activated partial thromboplastin time, $121 \mathrm{~s}$; and INR, $>6$.

Contrast-enhanced CT (• Fig. 7 and - Fig. 8) was performed and showed circumferential thickening of the jejunum and dilatation of the proximal intestinal segments.

Because of worsening of the symptoms and development of complete intestinal obstruction in spite of conservative therapy, the patient underwent laparotomy with resection of the affected intestinal segment. Histolopathological examination showed hemorrhage and necrosis of the jejunal segment. The postoperative period was uneventful and the patient was discharged 12 days after admission.

\section{Discussion}

\section{Epidemiology}

Spontaneous intestinal intramural hematoma is a rare complication under anticoagulant therapy. Better et al. reported an incidence of 1 in 2,500 patients receiving warfarin [10]. The incidence is higher in males and the average age at presentation is 57.6 years [9].

Because of the rarity of spontaneous intramural small-bowel hematoma, there are only case reports dealing with this clinical entity. The largest series included 13 patients, of whom 8 received oral anticoagulation [7].

\section{Etiology}

Intramural small-bowel hematoma may arise spontaneously or after trauma. Blunt abdominal trauma is by far the most common etiologic factor for duodenal hematoma.

Med Klin Intensivmed Notfmed 2013 • 108:144-148 DOI 10.1007/s00063-012-0184-0

c) Springer-Verlag Berlin Heidelberg 2012

\section{A. Abdel Samie · R. Sun · A. Huber · W. Höpfner · L. Theilmann Spontaneous intramural small-bowel hematoma secondary to anticoagulant therapy: a case series}

\section{Abstract}

Spontaneous small-bowel hematomas most commonly involve the jejunum, followed by the ileum and duodenum, and occur in patients who receive excessive anticoagulation with phenprocoumon/warfarin or who have additional risk factors for bleeding.

We report three cases of intramural smallbowel hematoma, all complications of treatment with phenprocoumon, which nowadays is used extensively for therapeutic and prophylactic purposes.

Diagnosis can be readily attained by sonography and confirmed using computed tomography. Early diagnosis is crucial because most patients can be treated successfully without surgery.

Based on this experience and data from the literature, conservative treatment is recommended for intramural intestinal hematomas, when other complications needing laparotomy have been excluded.

Keywords

Intestinal hematoma - Phenprocoumon . Anticoagulant therapy · Small bowel · Case series

\section{Spontanes intramurales Dünndarmhämatom nach Antikoagulanzientherapie: Fallserie}

\section{Zusammenfassung}

Spontane Dünndarmhämatome betreffen vor allem das Jejunum, gefolgt vom lleum und Duodenum und treten bei Patienten unter exzessiver Antikoagulation mit Phenprocoumon/Warfarin beziehungsweise bei Patienten mit anderen Blutungsrisikofaktoren auf.

Wir berichten über 3 Patienten mit intramuralen Dünndarmhämatomen als Komplikation einer Phenprocoumontherapie, die heutzutage sehr breit für therapeutische und prophylaktische Zwecke eingesetzt wird.

Die Diagnose eines intramuralen intestinalen Hämatoms kann sonographisch gestellt und computertomographisch bestätigt werden. Eine Frühdiagnose ist essenziell, da die meisten Patienten erfolgreich konservativ behandelt werden können.

Aufgrund unserer Erfahrung und der bisher publizierten Fallberichte wird nach Ausschluss von Komplikationen, die einer chirurgischen Intervention bedürfen, ein konservatives Vorgehen bei intramuralen intestinalen Hämatomen empfohlen.

Schlüsselwörter Intestinales Hämatom · Phenprocoumon . Antikoagulanzientherapie - Dünndarm . Fallserie
The relatively fixed retroperitoneal position of the duodenum and its close anterior relationship to the lumbar spine renders it susceptible to blunt trauma of a crushing type and accounts for the higher incidence of duodenal involvement in this situation [1].

This condition involves mainly children, and the trauma is frequently insignificant and forgotten by the patient. The weak abdominal muscles and the high costal margin contribute to the susceptibility of children to intramural duodenal hematoma [11].

Over-anticoagulation with warfarin is the most common cause of spontaneous intramural small-bowel hematoma in adults. In addition to excessive anticoagulation, this condition has also been described as a complication of bleeding disorders, malignancies, and vasculitis [7].

Excessive anticoagulation with an INR greater than 6 was associated with developing this rare complication in our 3 patients, who all received phenprocoumon because of chronic atrial fibrillation.

Although spontaneous intramural small-bowel hematoma might be expected to occur with over-anticoagulation by heparin, we found only one case report describing this complication in a child who received therapeutic doses of lowmolecular-weight heparin because of deep venous thrombosis [12]. 


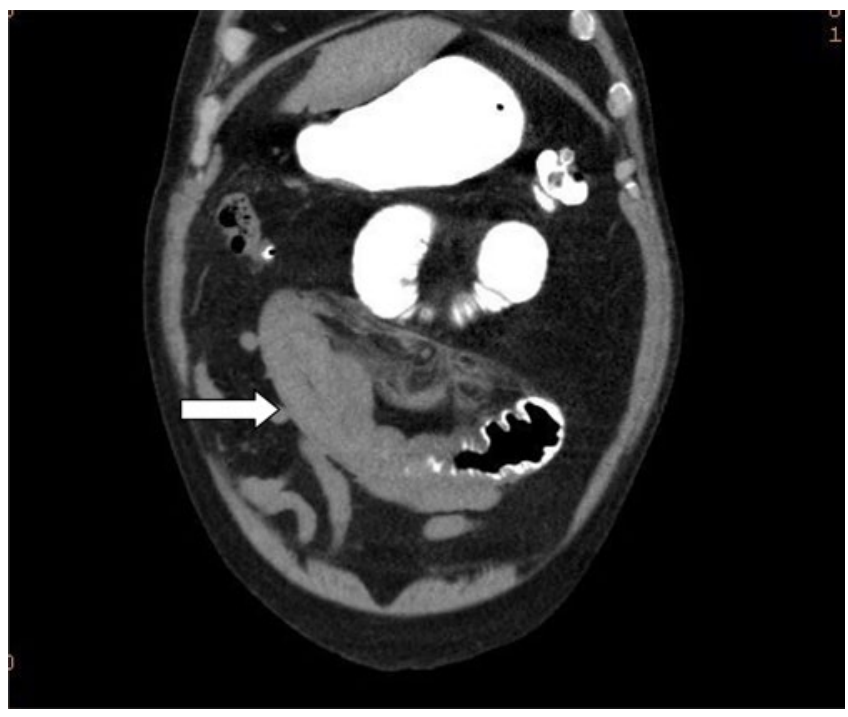

Fig. $7 \Delta$ Contrast-enhanced CT showing circumferential thickening of the jejunum (arrow) and dilatation of the proximal intestinal segments

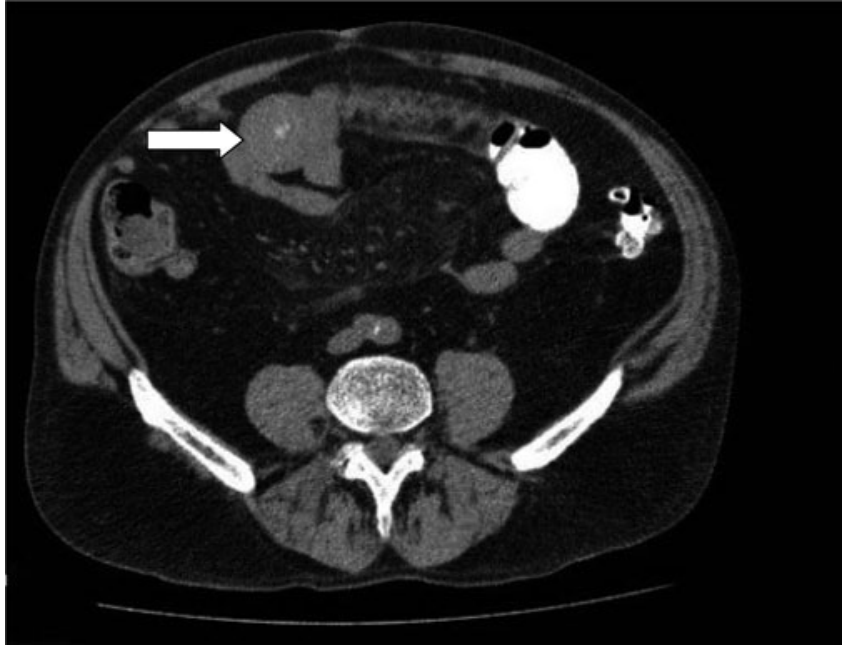

Fig. $8 \Delta$ Contrast-enhanced CT showing circumferential thickening of the jejunum (arrow) and dilatation of the proximal intestinal segments
The small bowel is affected in up to $85 \%$ of cases of spontaneous intramural gastrointestinal tract hematoma in patients under anticoagulation. Nevertheless, it remains unclear why the jejunum is the site of predilection for spontaneous hematoma.

Bleeding originates in the submucosa from small vessels. Some authors speculate that the progression of the symptoms is due to an intramural osmotic gradient caused by the hematoma, leading to expansion of the intestinal wall [13].

Small-bowel hematomas can extend into the colon; however, isolated cases are very rare. It has been hypothesized that the taeniae coli may play a protective role against the initiation or expansion of the hemorrhage [8].

\section{Clinical presentation}

The spectrum of presentation is wide and can vary from mild, vague abdominal pain to intestinal obstruction and an acute abdomen. Nausea and vomiting are found in half of the cases and are related to high intestinal obstruction involving the duodenum and proximal jejunum.

Patients may also present with gastrointestinal hemorrhage as a result of ruptured hematoma. However, obvious bleeding is present in less than half of the cases and cannot be relied upon as a diagnostic criterion [14]. Signs of perito- neal irritation may be present in some cases and usually indicate the development of complications, such as necrosis, perforation, or hemoperitoneum.

The average time from the appearance of symptoms until medical attendance is 2.5 days. The duration of anticoagulation therapy is 25.4 months on average [9].

In the largest reported series of spontaneous small-bowel hematoma involving 13 patients, the mean age of presentation was 64 years. Small-bowel obstruction was present in $85 \%$ of the patients, and the jejunum was the most common site of the hematoma, followed by the ileum and duodenum. All patients presented with abdominal pain. The mean INR at presentation was 11.6 [7].

In our patients, the mean age of presentation was 79 years and the average time from the beginning of symptoms until seeking medical help at the emergency department was 2.5 days. The jejunum was affected in two cases and the duodenum in one case. Abdominal pain was the leading symptom at presentation in all patients. All patients were under long-term anticoagulant therapy because of chronic atrial fibrillation with an INR greater than 6.

\section{Imaging findings}

CT is the key for diagnosis of this condition. Some authors have suggested that noncontrast CT should be performed before oral and intravenous contrast medium application, as contrast-enhanced CT alone may mask the presence of intramural hemorrhage [9]. Characteristic findings on CT include circumferential wall thickening, intramural hyperdensity, luminal narrowing, and intestinal obstruction $[7,15]$.

The sonographic appearance of acute intramural small-bowel hematoma consists of a thickened intestinal wall involving mainly the submucosal layer. However, this abnormality is not specific for intestinal tract hematomas and can be seen in a wide spectrum of disorders $[1,16]$.

The involved bowel segments appear longer in spontaneous hematomas than in traumatic hematomas with an average length of $23 \mathrm{~cm} \mathrm{[7,8].}$

The combination of CT and US can show the exact pathology in all patients, as demonstrated by some authors [17].

Wall thickening on CT may be also found in other conditions, including malignancy and inflammatory and ischemic bowel disease. However, in contrast to the latter, complete normalization of the imaging findings usually occurs within 2 months after the onset of symptoms.

In all three cases described in this paper, CT (contrast-enhanced CT in two cases and noncontrast CT in one case due to renal impairment) demonstrated circumferential wall thickening and luminal 
narrowing. The estimated length of the affected jejunal segment was $25 \mathrm{~cm}$ in one case and $20 \mathrm{~cm}$ in the other.

Sonography was performed in two cases and showed heterogeneous thickening of the intestinal wall.

The MRI characteristics of duodenal hematoma include a well-defined concentric ring of high signal intensity (ring sign) in the subacute bleeding. The unique MRI tissue characteristics (ring with short T1 and long $\mathrm{T} 2$ relaxation time) are attributable to the paramagnetic properties of iron in the hemoglobin degradation products within the hematoma [18].

However, no data are available on the imaging characteristics of small-bowel hematomas other than those that occur in the duodenum.

In case one described in this paper, the "ring sign" was detected on MRI, a finding which helped established the diagnosis of duodenal hematoma.

With the help of pull and push enteroscopy, deep small-bowel lesions can be visualized [19]. However, the role of endoscopy in the diagnosis of intramural hematoma is not well established, as almost all cases are diagnosed "noninvasively" with CT and US. Nevertheless, endoscopy may play a therapeutic role, as shown by Kwon et al. [20], who have successfully treated a duodenal hematoma with a needle-knife incision relieving obstructive symptoms.

\section{Therapy}

Owing to the rarity of this entity, there are no studies that contain sufficient evidence to standardize treatment.

The first step in the treatment of acute intramural small-bowel hematoma is discontinuation of the anticoagulant medication and correction of coagulation parameters with vitamin $\mathrm{K}$.

Conservative treatment usually leads to improvement of symptoms within 4-6 days. Complete resolution usually occurs within 2 months after the onset, because the hemorrhagic bowel is not necrotic and complete restitution occurs in almost all cases under conservative management [14, 21, 22].

It appears safe to resume anticoagulant therapy in patients after resolution of the hematoma as long as it is administered within the therapeutic range. Neither recurrence of intestinal obstruction or hematoma nor long-term sequelae were observed in this group [10].

Laparotomy is not indicated in uncomplicated intramural hematoma of the small bowel. Surgical exploration is reserved for cases with uncertain diagnosis or late complications, which include intra-abdominal hemorrhage, suspected ischemia, perforation, peritonitis, and intestinal obstruction not responding to conservative strategies $[9,10,14,22]$.

Two of our three patients were successfully treated conservatively. In the third case, laparotomy was performed owing to worsening of symptoms and signs of peritoneal irritation. In this case, complicating intestinal necrosis indicated resection of the affected hemorrhagic segment.

\section{Conclusion}

The recognition of intramural intestinal hematoma induced by anticoagulant drugs as a clinical entity is vitally important in diagnosis and treatment of this condition. A high index of suspicion of this rare complication of over-anticoagulation is required for making an accurate diagnosis.

Intramural hematoma of the small bowel should be searched for in any patient with abdominal pain who is receiving anticoagulant therapy, especially if the INR is excessively prolonged. It is extremely important to recognize this condition in order to avoid an unnecessary operation, since the outcome is usually excellent after conservative treatment.

\section{Corresponding address}

\section{A. Abdel Samie MD, FEBGH, FEFIM}

Department of Gastroenterology, Pforzheim Hospital

Kanzlerstr. 2-6, 75175 Pforzheim

abdelsamie@ngi.de

Conflict of interest. On behalf of all authors, the corresponding author states that there are no conflicts of interest.

\section{References}

1. Altikaya N, Parlakgüms A, Demir S, Yildirim T (2011) Small bowel obstruction caused by intramural hematoma sencondry to warfarin therapy: a report of two cases. Turk J Gastroenterol 22(2):199-202

2. McLauchlan J (1883) Fatal false aneurysmal tumour occupying nearly the whole of the duodenum. Lancet 2:203-205

3. Sutherland GA (1904) Intussusception and Henoch's purpura. Br J Dis Child 1:23-28

4. Liu Y, Yang S, Tong Q (2011) Spontaneous intramural hematoma of colon.Clin Gastroenterol Hepato(Epub)

5. Jarry J, Biscay D, Lepront D et al (2008) Spontaneous intramural haematoma of the sigmoid colon causing acute intestinal obstruction in a haemophiliac: report of a case. Haemophilia 14(2):383-384

6. Shimodaira M, Nakajima Y, Akiyama T, Koyama S (2011) Spontaneous intramural haematoma of the oesophagus. Intern Med J 41(7):577-578

7. Abbas MA, Collins JM, Olden KW (2002) Spontaneous intramural small bowel hematoma: imaging findings and outcome. Am J Roentgenol 179(6):1389-1394

8. Abbas MA, Collins JM, Olden KW, Kelly KA (2002) Spontaneous intramural bowel hematoma: clinical presentation and long germ outcome. Arch surg 137:306-310

9. Sorbello MP, Utiyama EM, Parreira JG et al (2007) Spontaneous intramural small bowel hematoma induced by anticoagulant therapy: review and case report. Clinics 62(6):785-790

10. Jimenez J (1999) Abdominal pain in a patient using warfarin. Postgrad Med J 75(890):747-748

11. Jones WR, Hardin WJ, Davis JT, Hardy JD (1971) Intramural hematoma of the duodenum. Ann Surg 173(4):534-544

12. Shaw PH, Rananathan S, Gaines B (2005) A spontaneous intramural hematoma of the bowel presenting as obstruction in a child receiving low-molecular-weight heparin. J Pediatr Hematol Oncol 27(10):558-560

13. Judd DR, Taybi H, King H (1964) Intramural hematoma of the small bowel; a report of two cases and a review of the literature. Arch Surg 89:527-535

14. Askey JM (1966) Small bowel obstruction due to intramural hematoma during anticoagulant therapy. Calif Med 104(6):449-453

15. Cheng J, Vemula N, Gendler S (2008) Small bowel obstruction caused by intramural hemorrhage. Secondary to anticoagulant therapy. Acta Gastroenterol Belg 71(3):342-344

16. Rauh P, Uhle C, Ensberg D et al (2008) Sonographic characteristics of intramural bowel hematoma. J Clin Ultrasound 36(6):367-368

17. Polat C, Dervisoglu A, Guven H et al (2003) Anticoagulant-induced intramural intestinal hematoma. Am J Emerg Med 21(3):208-211

18. Abdel Samie A, Sun R, Theilmann L (2009) A rare cause of obstructive jaundice. Gastroenterology 137(1):40, 394

19. Shinozaki S, Yamamoto H, Kita H et al (2004) Direct observation with double-balloon enteroscopy of an intestinal intramural hematoma resulting in anticoagulant ileus. Dig Dis Sci 49(6):902-905

20. Kwon C, Hyun KK, Kim HY et al (2009) Bowel obstruction caused by an intramural duodenal hematoma: a case report of endoscopic incision and drainage. J Korean Med Sci 24(1):179-183

21. Carkman S, Özben V, Saribeyoglu Ket al (2010) Spontaneous intramural hematoma of the small intenstine. Turkisch J Trauma Emerg Surg 16(2):165-169

22. Ashley S (1990) Spontaneous mesenteric hematoma and small bowel infarction complication oral anticoagulant therapy. J Royal Soc Med 83:116 